\title{
The effects of high and low meaningfulness and interitem association upon the level of difficulty of a serial task
}

\section{P. S. DELIN, DEPARTMENT OF PSYCHOLOGY, UNIVERSITY OF ADELAIDE, South Australia}

$A$ set of high and a set of low meaningful English words were each arranged in two different orders so as to maximize and to minimize association between adjacent items. The performance of Ss learning these lists suggests that, with these materials, adjacentitem association level is as powerful as meaningfulness in its effect upon list difficulty. These results support an item-associative view of the functional stimulus. Ancillary findings suggest list structure affects the strategies used by Ss.

The meaningfulness $(m)$ of individual items (as measured by the production method (Noble, 1952)), and closely related variables such as frequency and familiarity (Underwood \& Schulz, 1960) have long been regarded as potent variables affecting list difficulty. Deese (1959), showed that, in free recall, a measure of the degree of associative overlap of the list items was also strongly related to list difficulty. This variable has been further explored by Deese and others (e.g., Deese, 1962; Garskof \& Houston, 1963), but has usually been measured in terms of the extent to which pairs of items share associative hierachies (Deese, 1959, 1962), or of the frequency with which they elicit each other as free associations (Amster, 1967; Garskof et al, 1967). However, the use by Ss of natural language mediators (Montague \& Wearing, 1967; Martin \& Dean, 1966) makes it likely that factors other than associative overlap may be relevant in determining the "degree of relatedness" of a pair of items. Thus pairs like "whimper-chaos" may be much more highly related than their degree of associative overlap would suggest. This possibility lends some interest to the use of judges' ratings of degree of interitem association.

The use of such ratings would make it possible to present serial lists with the items arranged in different orders so as to achieve different average levels of interitem association without affecting any other major variable. This in turn would make it possible to explore the relation between meaningfulness and interitem association. Wearing \& Montague (1967) found that two paired associates lists which had been equated on most dimensions of meaningfulness were still not equally easy to learn, and that the difference appeared to be related to a difference in interitem association, as estimated by the numbers of natural language mediators produced by the Ss. The present study attempts to demonstrate this effect more directly.

Materials. Two sets of 12 words were selected from the list published by Saltz (1967) in such a way that one set had high $m$ (mean $m=8.6$ ) and the other had the lowest $m$ (mean $m=6.8$ ) that could be achieved consistent with all the words being nouns. In addition to being different in meaningfulness all words in the high $m$ set were at least 10 times more frequent than those in the low $m$ set.

The words in each set were next arranged in two different orders with the intention first of maximizing and then of minimizing the degree of association (a) between adjacent items. This was carried out by the $E$ on an intuitive basis. It was hoped that the next step in the preparation would validate this procedure.

The four word lists were broken down into their constituent word pairs (making 44 pairs), and these were presented in different random orders to 20 judges. The judges were instructed to indicate by placing a mark on an un-numbered scale running from "Very weak association" to "Very strong association" how strongly each pair of words seemed to them to be associated. These rating marks were then converted by measurement to scores rangirig from 0 to 10 . The split half reliability of the ratings was computed as rho $=.89$ Upon reassembly of the lists the two high $m$ lists were found to have mean $a$ indices of 2.64 and 7.06 , and the low $m$ lists gave means of 1.65 and 5.96 , there being practically no overlap in $a$ values between high $a$ and low $a$ lists. These findings were seen as justifying the subjectivity of the ordering procedure.

Method. Each of the four lists (High $m$, High $a(\mathrm{HH})$; High $m$, Low $a$ (HL); Low $m$, High $a(\mathrm{LH})$; Low $m$, Low $a(\mathrm{LL})$ ) was learned by 16 first year psychology students using the anticipation method. Presentation was by memory drum at a $4 \mathrm{sec}$ rate, and learning was carried to a criterion of one error-free anticipation of the list. Inter-trial interval was $10 \mathrm{sec}$. Each $S$ initially learned a practice list consisting of seven two-digit numbers, and the error scores in this task were used to achieve a rough matching of the Ss assigned to learn each experimental list. Four levels of learning ability were used in this matching procedure.

When the learning tasks had been completed all Ss were asked whether they had noticed anything particular about the experimental list, and also whether they had been aware of employing any particular technique in learning the list.

Results. The main results were as set out in Table 1. Because of the high degree of skew in the data, analyses of variance were carried out on logarithmic transformations of both errors to criterion and trials to criterion.

In the analysis of variance of errors both main variables and the interaction between them proved significant. Interitem association showed the most striking effect $(F=42.20, \mathrm{df}=1 / 60, p<.001)$, but meaningfulness was significant at the .025 level $(\mathrm{F}=5.68, \mathrm{df}=$ $1 / 60)$ and interaction at the .05 level $(F=4.59, \mathrm{df}=1 / 60)$. HH was the easiest list, followed by $\mathrm{LH}, \mathrm{HL}$, and finally $\mathrm{LL}$. The differences $\mathrm{HH} / \mathrm{LH}$ and $\mathrm{LH} / \mathrm{HL}$ were significant at the .02 level, using $t$ tests on the transformed scores $(t=2.65$ and $t=2.66)$, but the $\mathrm{HL} / \mathrm{LL}$ difference was insignificant $(t=.97)$. In a similar analysis of trials to criterion both main effects were significant (meaningfulness $F=4.60, p<.05$, interitem association $F=$ $27.83, \mathrm{p}<.001$ ), but there was no interaction. The ordering of the lists was the same, but the only difference significant according to the $t$ test was that between $\mathrm{LH}$ and HL $(t=2.16$, $p<.05)$. The other differences would, however, have reached significance if one-tailed tests had been used.

The answers to the questions asked after the learning session showed some striking intergroup differences. Thus all but three HH Ss noticed that all items were interconnected, and most of them based their learning on this. The LH Ss tended to notice particular pairings as connected, and to supplement these connections by the use of mixed strategies involving similarities in word shape, spelling, and meaning. Neither HL nor LL Ss showed much awareness of the experimental arrangement of the lists. Rote learning (without much reference to meaning) was described by about half the members of both groups as the main technique used but Ss in LL showed greater awareness of remote associations, and several appeared to have made some use of these. Attempts to relate the meanings of adjacent words were more common in $\mathrm{HL}$, whereas LL Ss used a wide variety of techniques related to form rather than to content.

There was some suggestion in the data that the differences in list structure led to differences in both type and distribution of errors. Thus Ss with high meaningful lists made many more of

Table 1

Means, Medians and S.D.s for Untransformed Errors and Trials Data

\begin{tabular}{|c|c|c|c|c|c|c|c|c|}
\hline & \multicolumn{2}{|c|}{$\mathrm{HH}$} & \multicolumn{2}{|c|}{ HL } & \multicolumn{2}{|c|}{ LH } & \multicolumn{2}{|c|}{$\mathbf{L L}$} \\
\hline & Errors & Trials & Errors & Trials & Errors & Trials & Errors & Trials \\
\hline Mean & 4.1 & 2.8 & 24.3 & 6.2 & 8.5 & 4.6 & 25.5 & 7.0 \\
\hline S.D. & 4.56 & 1.58 & 25.62 & 4.16 & 6.56 & 2.93 & 16.80 & 3.00 \\
\hline Median & 2.5 & 2.5 & 13.5 & 5.0 & 7.0 & 4.0 & 17.0 & 6.5 \\
\hline
\end{tabular}


their errors in the form of intralist intrusions than of omissions, whereas the reverse was true of Ss learning the low meaningfulness lists. The HL Ss made in absolute terms more intrusion errors than did the LL Ss although the difference in mean total errors was in the opposite direction. Similarly, there was a marked difference between high and low interitem association groups in the proportion of all errors which occurred after one correct anticipation of the item in question had been made. Thus expressing these errors as fractions of the total errors for each group $\mathrm{HH}$ made $17 / 66$ errors of this type while LH made $12 / 136$. The equivalent figures for the other two groups were $113 / 389$ for $\mathrm{HL}$ and $119 / 408$ for LL.

Given the differences in total performance between the groups it would be difficult to assess the significance of these post hoc observations, but they certainly look as if they would warrant more direct examination in experiments designed for the purpose.

Discussion and Conclusions. The results of the analyses of variance show that both meaningfulness and interitem association (in terms of judges' ratings of the degree of relatedness of word pairs) affect the level of difficulty of serial lists of English nouns. Thus the degree of ease with which a list is learned may be as much affected by the order of the items in the list as by considerations of what the items are.

The fact that the same list of words may be either more or less difficult to learn if arranged in a different order has implications for theories about the nature of the functional stimulus. Thus it would be difficult to account for this phenomenon if it was assumed that associative links with other items in the list played no part in serial learning.
The less formal findings in this study lend some support to the view that the structure of the list has some determining effect both on the strategy that a $S$ will adopt and on the type and distribution of the errors he makes. The relations between these findings, as well as their validation, are topics for future research.

\section{REFERENCES}

AMSTER, H. Convergent association norms for ten-year-old children and college age adults. Psychon. Monogr. Suppl., 1967. Vol. 2, No. 1 (Whole No. 17).

DEESE, J. Influence of inter-item associative strength upon immediate free recall. Psychol Rep., 1959, 5, 305-312.

DEESE, J. On the structure of associative meaning. Psychol. Rev., 1962, 69, 161-175.

GARSKOF, B. E., \& HOUSTON, J. P. The measurement of verbal relatedness: an idiographic approach. Psychol. Rev., 1963, 70, 277-288.

GARSKOF, B. E., HOUSTON, J. P., \& MEDNICK, S. A. Overlap and direct associative strength norms for 480 word pairs. Psychon. Monogr. Suppl, 1967, Vol. 2, No. 2 (Whole No. 18).

MARTIN, R. B., \& DEAN, S. J. Reported mediation in paired-associate learning. J. verbal Learn. verbal Behav., 1966, 5, $23-27$.

MONTAGUE, W. E., \& WEARING, A. J. The complexity of natural language mediators and its relation to paired associate learning. Psychon. Sci, 1967, 7, 135-136.

NOBLE, C. E. An analysis of meaning. Psychol. Rev., 1952, 421-430.

WEARING, A. J., \& MONTAGUE, W. E. Associability of CVC-word pairs and its relation to list difficulty. Psychon. Sci., 1967, 7, 133-134. 